\title{
Coherent functional expansions in quantum field theory
}

\author{
P. D. Drummond \\ Centre for Quantum and Optical Science, Swinburne University of Technology, \\ Melbourne 3122, Australia \\ Kavli Institute for Theoretical Physics, UC Santa Barbara, USA.
}

\begin{abstract}
A new formalism is introduced to treat problems in quantum field theory, using coherent functional expansions rather than path integrals. The basic results and identities of this approach are developed. In the case of a Bose gas with point-contact interactions, this leads to a soluble functional equation in the weak interaction limit, where the perturbing term is part of the kinetic energy. This approach has the potential to prevent the Dyson problem of divergence in perturbation theory.
\end{abstract}

\section{Introduction}

One of the most difficult problems in theoretical physics is conceptually very simple. How does one calculate the ground state of an interacting many-body quantum system? Analytic solutions do not usually exist in more than one space dimension, and a finite computer cannot easily solve such exponentially hard problems [1]. Conventional path integrals [2], are not exactly soluble when there are interactions. Infinite order perturbation theory with truncated sets of diagrams is frequently used to handle strongly interacting systems. Approaches like this are being tested in experiments in ultra-cold atomic physics [3, 4] using Feshbach resonances that can reach strongly interacting, universal behavior [5], [6, 7].

However, as first pointed out in the work of Dyson [8], conventional perturbation theory does not converge as a power series in the coupling constant [9, 10]. This cannot be cured using renormalization [11]. Similar convergence issues exist in other quantum theories [12]. They occur when the exact theory is not analytic at zero coupling; an example is the quantum anharmonic oscillator 13. While other methods exist for Bose condensates [14, 15, 16, 17, any attempt to use the interaction term as a perturbation is likely to have divergences for this reason [1]. 
Here an approach is introduced in which coherent functional states are used to treat a non-relativistic quantum field theory. This allows one to treat the weakly interacting ground state in a Bose condensate, where the interaction term is included in the unperturbed part of the problem.

The functional expansion used is an integral over amplitudes of coherent states. Related stochastic techniques [18] and variational methods [19] are known. Here we show that in the limit of weak interactions, one can define an asymptotic ground state which includes the nonlinear coupling. This leaves part of the kinetic energy as a perturbation, rather than the interaction term. Identities and functional equations are obtained in this Letter, together with limiting behaviour, and more detailed properties will be treated elsewhere.

\section{Coherent states and phase integrals}

The notion of a coherent state has proved useful in understanding the basis of coherence [20, 21]. An unnormalized functional coherent state [22, 21, 23, 24] of a bosonic quantum field $\hat{\Psi}(x)$ is

$$
\| \alpha\rangle \equiv \exp \left(\int_{V} \alpha \cdot \hat{\Psi}^{\dagger} d x\right)|0\rangle,
$$

with a coherent amplitude field $\alpha(x)$, where $|0\rangle$ is the vacuum state. The integration is over a space coordinate $x$ in a $D$ dimensional real vector space, volume $V$, with periodic boundary conditions and volume measure $d x$. Arguments of functions inside space integrals are omitted. Commutators for the scalar fields are $\left[\hat{\Psi}(x), \hat{\Psi}^{\dagger}\left(x^{\prime}\right)\right]=\delta^{D}\left(x-x^{\prime}\right)$. The coherent states are not orthogonal, since [25, 26, 27]:

$$
\left\langle\alpha \| \alpha^{\prime}\right\rangle=e^{G\left[\alpha^{\prime}, \alpha^{*}\right]}
$$

where $G\left[\alpha, \alpha^{\prime}\right]=\int_{V} \alpha^{*} \cdot \alpha^{\prime} d x$. Introducing a normalizing factor $G \equiv G[\alpha, \alpha]$, the normalized functional coherent state is: $|\alpha\rangle \equiv \exp [-G / 2] \| \alpha\rangle$.

Arbitrary quantum states can be written as functional expansions $|f[\alpha]\rangle$ and phase-space representations over coherent states [21, 28, 29, 24]. These are defined here using the unnormalized coherent states, with a functional measure $d[\alpha]$ such that:

$$
\left.|f[\alpha]\rangle_{\alpha} \equiv \int f[\alpha] \| \alpha\right\rangle d[\alpha]
$$

With number conserving Hamiltonians, it is natural to use a basis of numberprojected coherent states, $\| \alpha\rangle_{N}[30$, which are obtained through projection onto a particle number $N$ with a projection operator $\hat{P}_{N}$, using a phase integral [31],

$$
\left.\left.\| \alpha\rangle_{N} \equiv \hat{P}_{N} \| \alpha\right\rangle=\int \frac{d \phi}{2 \pi} e^{-i \phi N} \| \alpha e^{i \phi}\right\rangle
$$


This is shown to be a number projection by a Taylor expansion of the exponential in (11). As a result,

$$
\| \alpha\rangle_{N} \equiv \frac{1}{N !}\left(\int_{V} \alpha \cdot \hat{\Psi}^{\dagger} d x\right)^{N}|0\rangle
$$

The inner product of $\| \alpha\rangle_{N}$ and $\left.\| \alpha^{\prime}\right\rangle_{N^{\prime}}$ vanishes unless $N=N^{\prime}$. One can similarly show that the inner product of number-conserving coherent states is $\left\langle\alpha \| \alpha^{\prime}\right\rangle_{N}=G^{N}\left[\alpha, \alpha^{\prime}\right] / N$ ! , by expanding the resulting exponential in a power series in $G\left[\alpha, \alpha^{\prime}\right]$.

\subsection{Coherent phase fields}

It is useful to employ a phase field notation, such that $\alpha(x)=\exp (\theta(x)+i \phi(x))$, where $\phi(x), \theta(x)$ are phase and amplitude fields. Here $\theta(x)=\ln |\alpha(x)|=$ $\ln r(x)$ determines the coherent logarithmic amplitude. A phase integral by itself is known to give a complete expansion for any system with a finite particle number per mode [32, so for simplicity we now assume that $r$ is a constant. The inner products of two coherent functional states are

$$
\left\langle\alpha \| \alpha^{\prime}\right\rangle=\exp \left(\int_{V} r r^{\prime} e^{i\left(\phi^{\prime}-\phi\right)} d x\right)=K\left[\phi, \phi^{\prime}\right] .
$$

Similarly, the inner products for the number-projected case are:

$$
\left\langle\alpha \| \alpha^{\prime}\right\rangle_{N}=\frac{1}{N !}\left[\int_{V} r r^{\prime} e^{i\left(\phi^{\prime}-\phi\right)} d x\right]^{N}=K_{N}\left[\phi, \phi^{\prime}\right] .
$$

\subsection{Coherent phase functional states}

One can also write the quantum field state functional integral in the form:

$$
\left.|\psi\rangle \equiv \int \psi[\phi] \| \alpha\right\rangle d[\phi]
$$

where the Jacobian for this measure change, $J=|d[\boldsymbol{\alpha}] / d[\phi]|$, is absorbed into the definition of $\psi[\phi]$. The following inner product holds:

$$
\left\langle\psi \mid \psi^{\prime}\right\rangle=\iint \psi^{*}[\phi] \psi^{\prime}\left[\phi^{\prime}\right] K\left[\phi, \phi^{\prime}\right] d[\phi] d\left[\phi^{\prime}\right] .
$$

\section{Differential identities}

Differential identities can be used to transform operator equations into functional differential equations. Coherent states are eigenstates of annihilation operators, so that $\hat{\psi}(x) \| \alpha\rangle=\alpha(x) \| \alpha\rangle$. From differentiating Eq (1), one obtains a differential identity for the creation operator:

$$
\left.\left.\hat{\psi}^{\dagger}(x) \| \alpha\right\rangle=\frac{\delta}{\delta \alpha(x)} \| \alpha\right\rangle .
$$


For systems with a conserved particle number, the Hamiltonian generates simple identities for number-conserving quadratic forms. Since the global projection operator $\hat{P}_{N}$ commutes with any number-conserving function of the field operators, the results below apply equally to number-projected states. Applying these identities to the particle density operator, $\hat{n}(x)=\hat{\psi}^{\dagger}(x) \hat{\psi}(x)$, one obtains that:

$$
\left.\hat{n}(x) \| \alpha\rangle=\frac{1}{i} \frac{\delta}{\delta \phi(x)} \| \alpha\right\rangle \text {. }
$$

For number-conserved states, $\left.\hat{N} \| \alpha\rangle_{N}=N \| \alpha\right\rangle_{N}$. In this case, it is useful to define a Fourier transform, $\phi(x)=\sum_{k} e^{i k \cdot x} \phi_{k}$, so the functionals are now scalar functions of the vector $\phi$. On defining $\partial_{k} \equiv \partial / \partial \phi_{k}$, the corresponding differential relationship is

$$
\frac{\delta}{\delta \phi(x)}=\frac{1}{V} \sum_{k} e^{-i k \cdot x} \partial_{k}
$$

For the number density operator, one can introduce an average particle density $\rho=N / V$ in order to replace the derivative over $\phi_{0}$, so that this can also be written as:

$$
\left.\hat{n}(x) \| \alpha\rangle_{N}=\left[\rho+\frac{1}{i V} \sum_{k \neq 0} e^{-i k \cdot x} \partial_{k}\right] \| \alpha\right\rangle_{N}
$$

\subsection{Functional identities}

To evaluate an observable or mean value property, consider an arbitrary operator O so that:

$$
\left.\hat{O}|\psi\rangle \equiv \int \psi[\phi] \hat{O} \| \alpha\right\rangle d[\phi]
$$

Any operator $\hat{O}$ can be transformed so that $\left.\hat{O}|\psi\rangle \equiv \int \| \alpha\right\rangle \mathcal{O}[\phi] \psi[\phi] d[\phi]$, where $\mathcal{O}$ is a differential operator acting on $\psi$. Hence, provided $|\psi\rangle$ is normalized, the expectation of an operator is:

$$
\operatorname{Tr}(\hat{\rho} \hat{O})=\iint \psi^{*}[\phi] K\left[\phi, \phi^{\prime}\right] \mathcal{O}\left[\phi^{\prime}\right] \psi^{\prime}\left[\phi^{\prime}\right] d[\phi] d\left[\phi^{\prime}\right] .
$$

The identities are obtained as follows. Firstly, the action of annihilation operators is simply:

$$
\left.\hat{\psi}(x)|\psi\rangle=\int \psi[\phi] \alpha(x) \| \alpha\right\rangle d[\phi]
$$

and for creation operators, after partial integration,

$$
\left.\hat{\psi}^{\dagger}(x)|\psi\rangle=\int \| \alpha\right\rangle \frac{i}{\alpha(x)} \frac{\delta \psi[\phi]}{\delta \phi(x)} d[\phi] .
$$


This can be iterated for any polynomial in field operators. For example, the particle density operator gives, after partial integration,

$$
\left.\hat{n}(x)|\psi\rangle=i \int \frac{\delta \psi}{\delta \phi(x)} \| \alpha\right\rangle d[\phi] .
$$

These differential identities are also applicable to the number projected coherent phase states, apart from the global phase derivative. This can be removed using the mapping $\partial_{0} \rightarrow\{-i N\}$, after partial integration. Terms like this will be added in braces to indicate a replacement can be made. Thus, the identities apply to either $\psi$ or to $\psi_{N}$, together with the substitution $\mathcal{O} \rightarrow \mathcal{O}_{N}, K \rightarrow K_{N}$

\section{Hamiltonian as a differential operator}

Next, consider a non-relativistic interacting Bose gas with a Hamiltonian of form $\hat{H}=\hat{H}^{K}+\hat{H}^{U}+\hat{H}^{I}$, where the energy includes a chemical potential [33], so that

$$
\begin{aligned}
\hat{H}^{K} & =-\frac{\hbar^{2}}{2 m} \int \hat{\psi}^{\dagger} \nabla^{2} \hat{\psi} d x, \\
\hat{H}^{U} & =\int(U-\mu) \hat{n} d x, \\
\hat{H}^{I} & =\iint \hat{n}(x) U^{I}\left(x-x^{\prime}\right) \hat{n}\left(x^{\prime}\right) d x d x^{\prime} .
\end{aligned}
$$

The ground state or energy eigenstate is a number eigenstate $|\psi\rangle_{N}$, since $\hat{N}$ and $\hat{H}$ commute. It is assumed that $U^{I}>0$, in order to have a stable, bounded Hamiltonian. The Hilbert space operators are now expressed using differential identities so that:

$$
\left.\hat{H}|\psi\rangle=-\frac{\hbar^{2}}{2 m} \int \| \alpha\right\rangle \mathcal{L}[\phi] \psi[\phi] d[\phi]
$$

where differential operators act on all terms to the right including the wavefunction $\psi$, and the ground state of $\hat{H}$ has the largest eigenvalue of $\mathcal{L}$.

\subsection{Differential identities for the Hamiltonian}

For the kinetic energy term, the coherent eigenvalue identity for the annihilation operator gives

$$
\left.\hat{H}^{K}|\psi\rangle=\frac{\hbar^{2}}{2 m} \int \| \alpha\right\rangle\left\{\int\left[\frac{1}{i \alpha} \nabla^{2} \alpha\right] \frac{\delta}{\delta \phi} d x\right\} \psi[\phi] d[\phi] .
$$

If the coherent amplitude is chosen as constant in space then the following choice is possible, although it is not the only one:

$$
\mathcal{L}^{K}=\int \frac{\delta}{\delta \phi}\left[\nabla^{2} \phi+i|\nabla \phi|^{2}\right] d x .
$$


To obtain an identity for an external potential energy term, set $u(x)=$ $2 m(U(x)-\mu) / \hbar^{2}$, and the simplest differential operator is:

$$
\mathcal{L}^{U}[\phi]=-i \int u \frac{\delta}{\delta \phi} \psi[\phi] d x,
$$

where as elsewhere, the position arguments of $u(x)$ are suppressed inside a space integral. In the case of an external potential with Fourier transform

$$
u(x)=\sum_{k} u_{k} e^{i k x},
$$

the differential operator for the corresponding energy term in the general case is $\mathcal{L}^{U}[\phi]=-i \sum_{k} u_{k} \partial_{k} \psi$, and for a number conserving expansion:

$$
\mathcal{L}_{N}^{U}[\phi]=-\left[N u_{0}+i \sum_{k \neq 0} u_{k} \partial_{k} \psi\right] .
$$

Defining $U^{I}(x)=\sum U_{k}^{I} \exp (i k x)$, the interaction Hamiltonian becomes:

$$
\begin{aligned}
\hat{H}^{I}|\psi\rangle & \left.=-\int \| \alpha\right\rangle \iint U^{I}\left(x-x^{\prime}\right) \frac{\delta^{2} \psi[\phi]}{\delta \phi(x) \delta \phi\left(x^{\prime}\right)} d x d x^{\prime} d[\phi] \\
& \left.=-\frac{g}{V} \| \alpha\right\rangle\left[\sum_{k} U_{k}^{I} \partial_{k} \partial_{-k}-\left\{N^{2} U_{0}^{I}\right\}\right] \psi d[\phi] .
\end{aligned}
$$

Setting $\gamma_{k}=2 m U_{k}^{I} /\left(\hbar^{2} V\right)$, the differential operator for the interaction term in the general case is:

$$
\mathcal{L}^{I}=V \iint \gamma\left(x-x^{\prime}\right) \frac{\delta^{2} \psi[\phi]}{\delta \phi(x) \delta \phi\left(x^{\prime}\right)} d x d x^{\prime}=\left[\sum_{k} \gamma_{k} \partial_{k} \partial_{-k}-\left\{\gamma_{0} N^{2}\right\}\right] \psi
$$

\subsection{Overall result}

The quantum field evolution can now be mapped into a differential equation with the form of a functional Fokker-Planck equation, such that:

$$
\frac{2 m}{i \hbar} \frac{\partial \psi}{\partial t}=-\mathcal{L} \psi
$$

This has the standard form of multi-variate Fokker-Planck linear operators, apart from the imaginary term. One can write this in Fourier components as

$$
\mathcal{L}=\sum_{k}\left[-\partial_{k} A_{k}+\gamma \partial_{k} \partial_{-k}\right]-\left(\bar{e}_{N}\right),
$$


where in the number conserved case the $k=0$ term is omitted, and the term $\bar{e}_{N}=N\left(u_{0}+\gamma N\right)$ is included instead. The first order drift term is:

$$
A_{k}=-k^{2} \phi_{k}+i\left(u_{k}-\sum_{q} q \cdot(k-q) \phi_{q} \phi_{k-q}\right) .
$$

For simplicity, we now assume an effective delta-function potential with a momentum cutoff, so $\gamma_{k}=\gamma$, giving a functional Fokker-Plank operator in phase:

$$
\mathcal{L}=\int\left[\frac{\delta}{\delta \phi}\left(\nabla^{2} \phi+i\left[|\nabla \phi|^{2}-u\right]\right)+\gamma V \frac{\delta^{2}}{\delta \phi^{2}}\right] d x .
$$

\section{Fokker-Planck equation}

In this section, the functional identities for the Hamiltonian are assembled and put to work. The goal is to understand the equivalent of the free-particle eigenstates. The limit of weak, but nonzero interactions is especially straightforward.

\subsection{Functional eigenvalue problem}

Using the identities derived above to write the energy eigenvalue problem in a functional form gives:

$$
\left.\hat{H}|\psi\rangle=E|\psi\rangle=-\frac{\hbar^{2}}{2 m} \int \mathcal{L}[\phi] \psi[\phi] \| \alpha\right\rangle d[\phi] .
$$

Defining the eigenvalue as $E=\hbar^{2} e /(2 m)$ the quantum field eigenvalue problem can be mapped into a functional calculus problem. It is simplest to define a chemical potential to shift the energy origin, so that the ground state has eigenvalue $e=0$, which defines an implicit condition on $\mu$. In the homogeneous case this has the general form of a Fokker-Planck equation, $\mathcal{L} \psi=\left[-\partial_{k} A_{k}+\gamma \partial_{k} \partial_{-k}\right] \psi=0$.

From the physics viewpoint, the meaning of the two terms is clear. The diffusion term, proportional to $\gamma$, is the coupling term which originates in the nonlinear interaction between the bosons. It results in noise-like diffusive behaviour. The drift terms $A_{k}$ originate in the single-particle kinetic and potential energy.

\subsection{Scaling behavior}

It is important to understand how the solutions behave in different limits, by introducing a scaled coupling $\gamma^{\prime}=\kappa \gamma$, and a corresponding Hamiltonian $\hat{H}^{\prime}$, where $\gamma$ is now used to define a reference interaction strength. The occupation number $N^{\prime}$ is varied such that $N^{\prime}=N / \kappa^{p}$, and the potential $u$ is taken to be uniform, such that $u^{\prime}=\kappa^{q} u$. Here $p, q$ are chosen so that so that the limit is physically nontrivial, while $N$ is a reference particle number. 
For $\psi(\phi)$ to have a well-defined limit, define a scaled phase variable $\phi \equiv$ $\phi^{(0)} / \kappa^{p}$, relative to an unscaled phase $\phi^{(0)}$. The total number of field degrees of freedom is held constant, and the changed functional measure is absorbed into the definition of $\psi$. The notation $|\psi\rangle^{\prime}$ now denotes a quantum state with a phase rescaling, where $|\psi\rangle^{\prime} \equiv \int \psi[\phi]\left|\phi \kappa^{p}\right\rangle d[\phi]$. From the calculus chain rule,

$$
\frac{\partial}{\partial \phi_{k}^{(0)}}=\frac{\partial}{\kappa^{p} \partial \phi_{k}} \text {. }
$$

Therefore, in the new coordinates and fields, $\hat{H}^{\prime}|\psi\rangle^{\prime}=\int \mathcal{L}^{\prime}[\phi] \psi^{\prime}[\phi]\left|\phi \kappa^{p}\right\rangle d[\phi]$, where the rescaled differential operator is now

$$
\mathcal{L}^{\prime}=\int \frac{\delta}{\delta \phi}\left[\nabla^{2} \phi+i\left(\kappa^{p}|\nabla \phi|^{2}+u \kappa^{q-p}\right)+\gamma V \kappa^{1-2 p} \frac{\delta}{\delta \phi}\right] d x
$$

\subsection{Weak coupling limit}

Consider a phase scaling transformation such that $\phi \equiv \phi^{(0)} / \sqrt{\kappa}$, and $p=q=$ $1 / 2$, which gives:

$$
\mathcal{L}^{\prime}=\int \frac{\delta}{\delta \phi}\left[\nabla^{2} \phi+i\left(\epsilon|\nabla \phi|^{2}+u\right)+\gamma V \frac{\delta}{\delta \phi}\right] d x .
$$

Here $\epsilon=\sqrt{\kappa}$, so for small $\kappa \rightarrow 0$, the correlated term in $|\nabla \phi|^{2}$ is negligible. Neglecting this, the Hamiltonian has a universal form, but it still includes the coupling term. Setting $\mathcal{L}^{\prime} \approx \mathcal{L}^{W}$ in this limit, the differential operator is:

$$
\mathcal{L}^{W}=\int \frac{\delta}{\delta \phi}\left[\nabla^{2} \phi+i u+\gamma V \frac{\delta}{\delta \phi}\right] d x
$$

With number conserving expansions, this can be rewritten in terms of momentum expansions as:

$$
\mathcal{L}_{N}^{W}=\sum_{k \neq 0} \frac{\partial}{\partial \phi_{k}}\left\{k^{2} \phi_{k}+\gamma \frac{\partial}{\partial \phi_{k}^{*}}\right\}-N\left(u_{0}+\gamma N\right)
$$

The ground state, number conserving solution of $\mathcal{L}_{N}^{W} \psi_{g}=0$ is a Gaussian in phase, with increasing phase-fluctuations at long wavelength, of form:

$$
\psi_{g}(\phi)=\exp \left[-\frac{1}{2 \gamma}\left(\sum_{k} k^{2}\left|\phi_{k}\right|^{2}\right)\right] .
$$

The corresponding physical energy per particle is $E / N=g \rho$, where $\rho=N / V$ is the mean particle density. This gives the usual mean-field energy in the absence of the kinetic correlation term, as one would physically expect for this limit of weak couplings and high density. 
A crucial issue is whether the unscaled term $i \epsilon|\nabla \phi|^{2}$, with $\epsilon=1$, can be used in a perturbation expansion around $\epsilon=0$. In QED, Dyson showed that perturbation theory cannot converge because the the exact theory is not analytic. It is discontinuous under a sign change in the charge. Here a sign change in $\epsilon$ cannot cause a discontinuity. It corresponds to conjugation with $\mathcal{L} \rightarrow \mathcal{L}^{*}$, which has no effect on the eigenvalue for a constant potential, and simply conjugates the eigenfunction. This is not yet a proof of analyticity or convergence, but it does show that instability under sign change of the perturbation is not present with this approach. While the differential operator $\mathcal{L}$ is not hermitian, this does not prevent the use of perturbation theory [34].

\section{Summary}

Here the weakly interacting ground state is the exact solution to a part of the Hamiltonian in differential notation, which already includes interactions. In this approach, the additional term required to obtain the full Hamiltonian is now a part of the kinetic energy. The perturbation does not include interactions, and there are no instabilities under a sign change of the perturbation term. As a result, this type of approach has the potential for a perturbation theory without Dyson's dilemma of nonconvergence.

\section{Acknowledgements}

This research was supported in part by the National Science Foundation under Grant No. NSF PHY-1125915, and by the Australian Research Council.

\section{References}

[1] Feynman R P 1982 Int. J. Theor. Phys. 21 467-488

[2] Feynman R P 1948 Rev. Mod. Phys. 20(2) 367-387

[3] Bloch I, Dalibard J and Zwerger W 2008 Rev. Mod. Phys. 80(3) 885-964

[4] Luo L, Clancy B, Joseph J, Kinast J and Thomas J 2007 Physical review letters $\mathbf{9 8} 080402$

[5] Ho T L 2004 Physical review letters 92090402

[6] Hu H, Drummond P D and Liu X J 2007 Nature Physics 3 469-472

[7] Hu H, Liu X J and Drummond P D 2010 New Journal of Physics 12063038

[8] Dyson F J 1952 Physical Review 85631

[9] Suslov I M 2005 Journal of Experimental and Theoretical Physics 100 $1188-1233$ 
[10] Boyd J P 1999 Acta Applicandae Mathematica 56 1-98

[11] Jaffe A 1965 Communications in Mathematical Physics 1 127-149

[12] Pernice S A and Oleaga G 1998 Phys. Rev. D 57(2) 1144-1158

[13] Zinn-Justin J 1996 Quantum Field Theory and Critical Phenomena International series of monographs on physics (Clarendon Press) ISBN 9780198518822

[14] Gardiner C W 1997 Phys. Rev. A 56(2) 1414-1423

[15] Castin Y and Dum R 1998 Phys. Rev. A 57(4) 3008-3021

[16] Morgan S A 2000 Journal of Physics B: Atomic, Molecular and Optical Physics 333847

[17] Gardiner S A and Morgan S A 2007 Phys. Rev. A 75(4) 043621

[18] Carusotto I, Castin Y and Dalibard J 2001 Phys. Rev. A 63(2) 023606

[19] He Q Y, Reid M, Opanchuk B, Polkinghorne R, Rosales-Zárate L and Drummond P 2012 Frontiers of Physics 7 16-30

[20] Schrödinger E 1926 Naturwissenschaften 14 664-666

[21] Glauber R J 1963 Phys. Rev. 131(6) 2766-2788

[22] Bargmann V 1961 Commun. Pure Appl. Math. 14 187-214

[23] Graham R and Haken H 1970 Zeitschrift für Physik 234 193-206

[24] Steel M J, Olsen M K, Plimak L I, Drummond P D, Tan S M, Collett M J, Walls D F and Graham R 1998 Phys. Rev. A 58(6) 4824-4835

[25] Campbell J 1896 Proceedings of the London Mathematical Society 1 381390

[26] Baker H 1902 Proceedings of the London Mathematical Society 34 347-360

[27] Hausdorff F 1906 Ber. Verh. Kgl. SÃ? chs. Ges. Wiss. Leipzig., Math.-phys. Kl. 58 19-48

[28] Chaturvedi S, Drummond P and Walls D F 1977 J. Phys. A 10 L187-L192

[29] Drummond P D and Carter S J 1987 J. Opt. Soc. Am. B 4 1565-1573

[30] Drummond P D and D R M 2016 ArXiv e-prints (Preprint 1610.01261)

[31] Aimi T and Imada M 2007 J. Phys. Soc. Jpn. 76084709

[32] Drummond P D and Gardiner C W 1980 J. Phys. A 13 2353-2368

[33] Fetter A and Walecka J 2003 Quantum Theory of Many-particle Systems Dover Books on Physics (Dover Publications) ISBN 9780486428277

[34] Sternheim M M and Walker J F 1972 Phys. Rev. C 6(1) 114-121 